Journal for ImmunoTherapy of Cancer Hui $R$, et al. Neoadjuvant immunotherapy for non-small cell lung cancer: right drugs, right patient, right time? Journal for ImmunoTherapy of Cancer 2021;9:e002248. doi:10.1136/ jitc-2020-002248

- Additional material is published online only. To view please visit the journal online (http://dx.doi.org/10.1136/jitc2020-002248).

Accepted 15 April 2021

Check for updates

(C) Author(s) (or their employer(s)) 2021. Re-use permitted under CC BY-NC. No commercial re-use. See rights and permissions. Published by BMJ.

For numbered affiliations see end of article.

Correspondence to

Dr Elizabeth Ahern;

elizabeth.ahern@monash.edu

\title{
Neoadjuvant immunotherapy for non- small cell lung cancer: right drugs, right patient, right time?
}

\author{
Elizabeth Ahern (D) , ${ }^{1,2,3}$ Ben J Solomon, ${ }^{4}$ Rina Hui, ${ }^{5,6}$ Nick Pavlakis, ${ }^{6,7}$ \\ Ken O'Byrne, ${ }^{8,9}$ Brett G M Hughes ${ }^{10,11,12}$
}

\section{ABSTRACT}

Standard curative treatment of early-stage non-small cell lung cancer (NSCLC) involves surgery in combination with postoperative (adjuvant) platinum-based chemotherapy where indicated. Preoperative (neoadjuvant) therapies offer certain theoretical benefits compared with adjuvant approaches, including the ability to assess on-treatment response, reduce the tumor bulk prior to surgery, and enhance tolerability in the preoperative setting. Indeed, the use of neoadjuvant therapies are well established in other cancers such as breast and rectal cancers to debulk the tumor and guide ongoing therapy, and neoadjuvant chemotherapy has similar efficacy but less toxicity in NSCLC. More recently, immune checkpoint inhibitors (ICI) targeting programmed death-1 (PD1)/PD1-ligand 1 (PDL1) have transformed the treatment of advanced NSCLC; the unique mechanisms of action of ICI offer additional rationale for assessment in the neoadjuvant setting. Preclinical studies in mouse cancer models support the proof of concept of neoadjuvant ICI (NAICI) through improvement of T-cell effector function and long-term memory induction. Preliminary early-phase human trial data support the proposition that NAICI in NSCLC may provide an feasible and potentially efficacious future treatment strategy and large, randomized phase III trials are currently recruiting to assess this approach. However, outstanding issues include defining optimal treatment combinations which balance high efficacy with acceptable toxicity, validating biomarkers to aid in patient selection, and avoiding potential pitfalls such as missing a window for successful surgery, that is, choosing the right drugs, for the right patient, at the right time. Predictive biomarkers to direct selection of therapy are required, and the validation of major pathological response (MPR) as a surrogate for survival will be important in the uptake of the neoadjuvant approach.

\section{INTRODUCTION}

Worldwide, lung cancer is the leading cause of cancer mortality with more than 1.7 million deaths in 2018 (18.4\% total cancer deaths worldwide). ${ }^{1}$ Non-small cell lung cancer (NSCLC) accounts for most lung cancer diagnoses (84\%). According to the Surveillance, Epidemiology and End Results database maintained by the National Cancer Institute (USA), 5-year survival for all patients with lung cancer is $19 \% .^{2}$ However, this varies by stage, with localized disease having $61 \%$ relative 5-year survival, falling to $35 \%$ for regional disease (spread to nearby structures or lymph nodes (LN)). NSCLC diagnosed at an advanced stage, where the cancer has spread to other parts of the body such as distant viscera, has a dismal 5 year prognosis at $6 \%$ survival. $^{2}$

Curative intent treatment approaches for early-stage disease often involve surgery, with other multimodality therapies recommended in certain settings to improve survival. Most commonly, chemotherapy and/or radiotherapy is administered in the adjuvant setting (postsurgery). ${ }^{3-5}$ Limited evidence suggests that chemotherapy given in the neoadjuvant setting (prior to surgery) is of comparable efficacy to adjuvant chemotherapy but with improved tolerability. ${ }^{5-8}$ Other proposed benefits of neoadjuvant chemotherapy (NACT) include increased likelihood to receive systemic treatment earlier to eradicate micrometastases, the ability to reduce tumor bulk prior to surgery potentially allowing more complete resections, and to directly observe the magnitude of pathological 'in vivo' regression which may aid in predicting subsequent outcome. On the other hand, a potential drawback of NACT is the risk of high-grade adverse events (AEs) which may impair the patient's ability to proceed onto definitive surgery. This is an important consideration given that, for all the advances in systemic therapy, surgery remains the single most effective treatment modality contributing to cure in patients presenting with operable cancer. ${ }^{9}$

More recently, immunotherapy comprizing immune checkpoint inhibitors (ICI) has changed the management of patients with advanced inoperable or metastatic NSCLC and has become part of the treatment paradigm for most patients with advanced disease 
(reviewed by Berghmans et $a l^{10}$ ). ICI has a distinct toxicity profile and potential patterns of response when compared with traditional cytotoxic chemotherapies. ${ }^{11} 12$ However, certain theoretical benefits could additionally derive from the administration of ICI in the neoadjuvant setting as opposed to the adjuvant use of this treatment postoperatively. In this view point, we review the emerging literature relating to preclinical and early-phase clinical trials of neoadjuvant ICI (NAICI) in NSCLC and discuss the pitfalls and future directions of this approach, in the context of ongoing large phase 3 NAICI trials.

\section{MANAGEMENT OF EARLY-STAGE NSCLC}

The improved survival for local and locoregional disease reflects that, in selected cases, management can be with curative intent-often with surgery as the mainstay of treatment. Surgery improves survival in early-stage disease: in a large retrospective cohort, those with stage I disease who were recommended but declined surgery had an estimated 5-year survival of $11 \%$, compared with $54 \%$ in those who underwent surgery. ${ }^{13}$ However, fewer than 30\% NSCLC patients receive surgery owing to factors such as advanced tumor stage at presentation or medical comorbidities. ${ }^{14-16}$ Surgery remains the gold standard of treatment for medically and surgically appropriate stage I-II NSCLC patients: in stage 1A-1B disease, 5 -year overall survival (OS) post-lobectomy ranges from $45 \%$ to $65 \%$ and declines in a stage-dependent manner thereafter. ${ }^{17}{ }^{18}$ Surgery is also recommended in selected patients with stage IIIA disease, often as part of multimodality therapy. ${ }^{19}$ Adjuvant chemotherapy is offered to patients with selected stage I-IIIA resected NSCLC and confers an additional absolute survival advantage of $5 \%$ at 5 years. ${ }^{34}$ The ADAURA trial reportedly demonstrated an impressive disease-free survival (DFS) benefit for osimertinib in resected epithelial growth factor receptor (EGFR)-mutated stage IB-IIIA NSCLC although OS outcomes are awaited. ${ }^{20}$

NACT, given prior to surgery, may provide benefits such as improved tolerability, improved likelihood of receiving treatment and ability to reduce tumor size preoperatively. In trials comparing neoadjuvant against adjuvant or perioperative chemotherapy, rates of chemotherapy completion were higher in the neoadjuvant arms, although with no differences in toxicity. ${ }^{67}$ However, it is not clear if cumulative adjuvant chemotherapy dose is associated with improved survival in lung cancer. ${ }^{21}$ In two prospective NACT trials, a meta-analysis and a large retrospective study, the benefit of NACT in NSCLC appears similar or non-superior to that of adjuvant chemotherapy in terms of DFS..$^{5-8}$ NACT can be part of the treatment paradigm for borderline-resectable or to downstage stage IIIA NSCLC patients as a bridge to surgery, for example in the presence of mediastinal LN metastases, although treatment decisions in this setting remain highly individualised. ${ }^{22}$
Neoadjuvant approaches bring a unique opportunity to assess pathologic responses via definitive surgery. Despite OS remaining the gold-standard endpoint, pathological response as a surrogate endpoint has been used in neoadjuvant trials in various cancer types. Pathological complete response (pCR), indicating no viable tumor remaining in the surgically resected specimen, has been correlated with OS in various malignancies following NACT, although the chance of achieving pCR is typically low. ${ }^{23}$ Adaptations of pathologic response rates include major pathological response (MPR), indicating $\leq 10 \%$ viable tumor cells remaining at surgical resection, or partial pathological response ( $\mathrm{pPR}, \leq 50 \%$ residual viable tumor), and these together with pCR have been variably assessed in NAICI trials in NSCLC and other cancers. ${ }^{23}$ MPR is prognostic in NSCLC following NACT as it is significantly correlated with improved survival. ${ }^{24-26}$ MPR has not yet been validated as a surrogate for survival in the setting of neoadjuvant immunotherapy, although MPR is accepted as a valid surrogate in neoadjuvant treatment of breast cancer, with Food and Drug Administration (FDA) approvals granted based on pathologic response rates for that disease. ${ }^{27}$

\section{PRECLINICAL RATIONALE FOR NAICI}

Preclinical models of cancer allow for an examination of immunological mechanisms underpinning NAICI. NAICI has been explored in various orthotopic mouse models of cancer, where neoadjuvant compared with adjuvant immunotherapy approaches have resulted in improved outcomes. ${ }^{28-30}$ NAICI was also examined in a syngeneic subcutaneous model of NSCLC, where neoadjuvant combination anti-PD1 plus anti-cytotoxic T-lymphocyteassociated protein 4 (CTLA4) resulted in superior metastasis-free survival compared with the same combination immunotherapy given in the adjuvant setting. ${ }^{31}$ Preclinical studies further demonstrated some common T-cell-mediated mechanisms of anti-tumor activity. These included significantly enhanced influx of tumorinfiltrating lymphocytes (TILs), an improved tumor antigen-specific T-cell response within the primary tumor and the suggestion of enhanced long-term tumor-specific immunological memory. As will be discussed, these findings are reminiscent of those reported in human trials of NAICI in NSCLC.

A recurrent role of innate immune system elements such as innate inflammatory signaling pathways and intact type one interferon responses, together with the requirement for cross-presenting dendritic cells (DCs), has been noted. These suggest a potential benefit of T-cell priming, perhaps in the tumor bed or locoregional secondary lymphoid organs, as is being increasingly recognized as essential for effective immunotherapy. ${ }^{32-35}$ Analysis of contributions by innate immune elements and DCs have not yet been presented in human NSCLC NAICI studies, although in a neoadjuvant trial of immunotherapy in melanoma (NCT02437279) ${ }^{36}$ low baseline 
Table 1 Reported studies involving neoadjuvant immunotherapy in resectable non-small cell lung cancer

\begin{tabular}{|c|c|c|c|c|}
\hline Trial name/identifier & Trial design & Neoadjuvant trial intervention & $\begin{array}{l}\text { Resection rate } \\
(\%)\end{array}$ & $\begin{array}{l}\text { MPR rate* } \\
(\%)\end{array}$ \\
\hline $\begin{array}{l}\text { TOP1201, } \\
\text { NCT01820754 }\end{array}$ & $\begin{array}{l}\text { Single arm, phase } 2, n=24, \text { Stage } \\
1 B-3 A \text { ( } 18 \text { with } N 2 \text { disease) }\end{array}$ & $\begin{array}{l}\text { Platinum-doublet NACT cycle } \\
\text { followed by NACT plus ipilimumab } \\
\text { (two further cycles) }\end{array}$ & 54 & NR \\
\hline $\begin{array}{l}\text { Forde et al, } \\
\text { NCT02259621 }\end{array}$ & $\begin{array}{l}\text { Single arm, phase } 2, n=21, \text { Stage } \\
1-3 A\end{array}$ & $\begin{array}{l}\text { Nivolumab } \\
\text { (two cycles) }\end{array}$ & 95 & 45 \\
\hline $\begin{array}{l}\text { LCMC3, } \\
\text { NCT02927301 }\end{array}$ & $\begin{array}{l}\text { Single-arm, phase } 2, n=181 \\
\text { Stage 1B-3B }\end{array}$ & $\begin{array}{l}\text { Atezolizumab } \\
\text { (two cycles) }\end{array}$ & 88 & 18 \\
\hline $\begin{array}{l}\text { Shu et al, } \\
\text { NCT02716038 }\end{array}$ & $\begin{array}{l}\text { Single arm, phase } 2, n=14 \text { (of } \\
\text { planned } 30 \text { ) }\end{array}$ & $\begin{array}{l}\text { Platinum-doublet } \\
\text { NACT+atezolizumab } \\
\text { (four cycles) }\end{array}$ & 87 & 57 \\
\hline NCT02904954 & $\begin{array}{l}\text { Randomized, phase } 2, n=34 \\
\text { Stage } 1-3 A\end{array}$ & $\begin{array}{l}\text { Arm-1: durvalumab } \\
\text { Arm-2: sub-ablative SBRT plus } \\
\text { durvalumab }\end{array}$ & 88 & $\begin{array}{l}\text { Arm-1: } 0 \% \\
\text { Arm-2: } 47 \%\end{array}$ \\
\hline
\end{tabular}

*MPR rate based on all reported patients, not only on those who underwent resection.

MRP, major pathological response; NACT, neoadjuvant chemotherapy; NR, not reported; SBRT, sub-ablative stereotactic radiotherapy.

tumorous expression of gene signature related to Batf3 ${ }^{+}$ (cross-presenting) DCs correlated with risk of relapse. ${ }^{37}$

\section{NEOADJUVANT IMMUNOTHERAPY TRIALS IN NSCLC}

The ability to conduct 'window-of-opportunity' intervention studies pre-surgical resection is an attractive setting for translational studies into the mechanism and efficacy of NAICI. Studies involving NAICI in resectable NSCLC where some outcomes have been reported are summarized in table 1 . The common findings include generally high resection rates, encouraging pathological regression rates and largely manageable toxicity profile. ${ }^{38-44}$ MPR has to date been the most common pathological response endpoint employed.

Forde et al published outcomes a in a phase II pilot study where 21 patients were treated with up to two doses of neoadjuvant nivolumab prior to planned resection of their tumors. ${ }^{38}$ Twenty-one patients were treated with nivolumab, but one was unresectable. The treatment was feasible and safe, without any treatment-related surgical delays, and with no previously unreported AEs. Only 23\% patients had $\mathrm{AE}$ of any grade, with one event being grade 3 or higher (grade 3 pneumonia where surgery was subsequently successfully performed). At 1-year postsurgery, $80 \%$ of resected patients were alive and without tumor recurrence. Impressively, theMPR rate was $45 \%(9 / 20)$, including three patients with pCR in the tumor bed (although one of these still had small residual tumor in a hilar node). No patients had evidence of progression, with a median of $65 \%$ pathological regression in the primary tumor. Radiological response did not correlate with pathological response, with $90 \%$ patients appearing to have stable disease per Response Evaluation Criteria in Solid Tumors, perhaps reflective of the early timepoint of follow-up imaging necessitated by planned surgery for all patients 4 weeks after commencing nivolumab as well as tumor infiltration of immune cells. Predicted tumor-associated neoantigens (TANA) were determined through transcriptomic analysis. TANA-specific T-cells were shown in one patient to expand significantly by the time of the second dose of neoadjuvant nivolumab and enter a contraction phase by the time of surgery, recapitulating findings described above in preclinical models. Using multiplex immunohistochemistry (IHC), the authors reported influx of TILs (comprizing lymphocytes and macrophages) in responding tumors. ${ }^{38}$ IHC correlates associated with effective anti-PD1 NAICI from participants in this trial were further elucidated. ${ }^{45}$ Three histopathological findings, comprizing immune activation (dense TIL infiltration with macrophages and tertiary lymphoid structures), massive tumor cell death, and tissue repair, were significantly enriched in MPR cases compared with non-MPR cases, and were also not present in any pretreatment specimens.

Two other phase 2 trials of NAICI have been recently updated. LCMC3 examined the impact of two cycles of neoadjuvant atezolizumab on MPR in stage IB-selected IIIB NSCLC patients and is the largest trial in lung 
cancer NAICI to date. ${ }^{39} 46$ Of the 181 reported patients, 159 had surgery (resection rate $88 \%$ ) and MPR rate was reported as $21 \%$ (including $7 \%$ pCR), excluding those resected patients who were subsequently found to have oncogenic driver mutations or rearrangements (none of which had MPR). A low rate of grade 3-4 treatmentrelated AEs (TRAE) was reported preoperatively $(6 \%)$ but two patients had grade 5 toxicity (both deemed nontreatment related). ${ }^{39}$ Interestingly, despite immature survival data, DFS and OS for stage I/II compared with stage III patients in LCMC3 appear similar and encouraging. At 1.5 years, $79 \%$ and $77 \%$ participants (stage I/ II and stage III, respectively), remain disease-free. ${ }^{46}$ NEOSTAR assessed the rate of MPR in patients treated with three neoadjuvant cycles of nivolumab alone $(\mathrm{N})$ or nivolumab plus one dose of ipilimumab with the first nivolumab cycle (NI) ${ }^{47}$ Forty-four patients with stage I-IIIA (single N2 positive node) NSCLC were recruited $(\mathrm{N}=23, \mathrm{NI}=21)$ and the outcome of 44 randomized patients was reported. Overall, 37 patients had surgery and of those, MPR rate was 29\% (N 22\%, NI 38\%). pCR rate was $29 \%$ in the NI cohort. Five high-grade (grade $3+$ ) immune-related TRAE were reported, including a case of grade 5 pneumonitis, which was seen in a patient treated with nivolumab alone. ${ }^{47}$ There was no apparent increase in toxicity, but more CD3+ T cell infiltration, CD8+ tissue resident and $\mathrm{CD} 4+$ effector memory $\mathrm{T}$ cells at surgery, and potentially greater induction of peripheral T-cell clonality and increased repertoire with neoadjuvant NI as compared with $\mathrm{N}$; these findings once again recapitulate those shown in preclinical models. At a median follow-up of 22 months, median recurrence-free survival and OS has not been reached in either cohort. ${ }^{47}$

Chemotherapy and radiotherapy can enhance antitumor immunity in various ways, including by inducing immunogenic cell death and modulating immune cells in the tumor microenvironment. ${ }^{48}$ Neoadjuvant antiPD1/PD-L1 chemoimmunotherapy in NSCLC has been explored in two phase 2 trials where data have been presented. MPR rates have been high $(57 \%-74 \%) .{ }^{414} 44$ One trial enrolled 30 current or former smokers with stage IB-IIIA resectable NSCLC to receive up to four cycles of neoadjuvant atezolizumab plus carboplatin and nab-paclitaxel. ${ }^{44}$ Twenty-six participants successfully underwent R0 surgical resection, of which 14 had a thoracotomy. Of the 17 participants with MPR (57\%), median DFS was 34.5 months compared with 14.3 months in those without MPR, but this did not reach statistical significance. Higher toxicity consistent with chemotherapy was seen, such as grade $3 / 4$ neutropenia rates of $50 \%$. Outcomes from the second trial, NADIM, assessing up to three cycles of neoadjuvant nivolumab plus carboplatin and paclitaxel followed by adjuvant nivolumab in 46 participants with resectable stage IIIA NSCLC, are also impressive. A 12-month progression-free survival (PFS) $(96 \%)$ and OS $(98 \%)$ was reported. ${ }^{41}$ Forty-one $(89 \%)$ participants had microscopically complete resection, and of those, at a median follow-up of 24.0 months, DFS was
$85 \%{ }^{49}$ Postoperative surgical complications were noted in 12 of $41(29 \%)$ resected participants such as infection or air leak. In this study, only $30 \%$ had grade 3 or higher toxicities of any type. Importantly, MPR achieved by 34 of $41(83 \%)$ participants who underwent resection was significantly associated with improved 24-month PFS compared with those not achieving MPR in a post hoc analysis $(88 \%$ vs $57 \%, \mathrm{p}=0.01){ }^{49}$ Overall, 26 of 46 total trial participants achieved pCR. ${ }^{49}$ In both chemoimmunotherapy studies, no surgical delays were noted owing to neoadjuvant treatment. ${ }^{44}$ Finally, NCT02904954 explored two cycles of durvalumab with or without subablative radiotherapy. ${ }^{50}$ Overall, eight patients of 34 (24\%) achieved MPR, all in the immunoradiotherapy arm. Preoperative high-grade toxicity rates (grade 3-4) were acceptable (12\%). Addition of radiotherapy modulated the tumor microenvironment through influx of immune cells including lymphocytes and DCs. ${ }^{50}$

Given these encouraging results, larger phase 3 randomized trials of neoadjuvant chemoimmunotherapy are currently recruiting (table 2) ${ }^{51-54}$ In these studies, the patient population has been refined to mainly include stage II-III (including some select IIIB) disease. In a press release, the trial sponsor has announced that CheckMate-816, assessing neoadjuvant nivolumab plus chemotherapy has met its primary endpoint of improved pCR compared with chemotherapy alone, but data are awaited.

\section{RIGHT DRUGS? EXPERIMENTAL STRATEGIES IN LUNG CANCER NAICI}

In addition to these ongoing neoadjuvant chemoimmunotherapy trials, ongoing smaller phase $1 / 2$ studies of NAICI in resectable NSCLC are exploring novel combinations and targets, including combinational checkpoint immunotherapy, chemoimmunotherapy, immunoradiotherapy, and even chemoimmunoradiotherapy (online supplemental table 1). Overall, these studies have a variety of primary endpoints mainly relating to safety and feasibility and/or assessment of tumor response or survival outcomes. Furthermore, the patient populations are heterogenous, with selection of locoregionally advanced patients and/or potentially resectable patients among trials combining different modalities (eg, chemo-ICI or immunoradiotherapy), but with earlier stage patients permitted (stage I-IIIA) in the NAICI monotherapy trials. ${ }^{5-59}$ Two trials are assessing novel immunotherapy combinations in stage I-IIIA NSCLC patients (combining anti-PD1 with anti-Lag3 or anti-RANKL) ${ }^{6061}$ Three studies are assessing neoadjuvant chemoimmunotherapy in stage III NSCLC patients, and these all include adjuvant ICI treatment postsurgery. ${ }^{62-64}$ Finally, two trials are assessing a potential role for neoadjuvant immunoradiotherapy in patients with stage III NSCLC, with one of these involving neoadjuvant chemoimmunoradiotherapy and adjuvant ICI. ${ }^{6566}$ 
Table 2 Ongoing phase 3 neoadjuvant chemoimmunotherapy trials in resectable non-small cell lung cancer

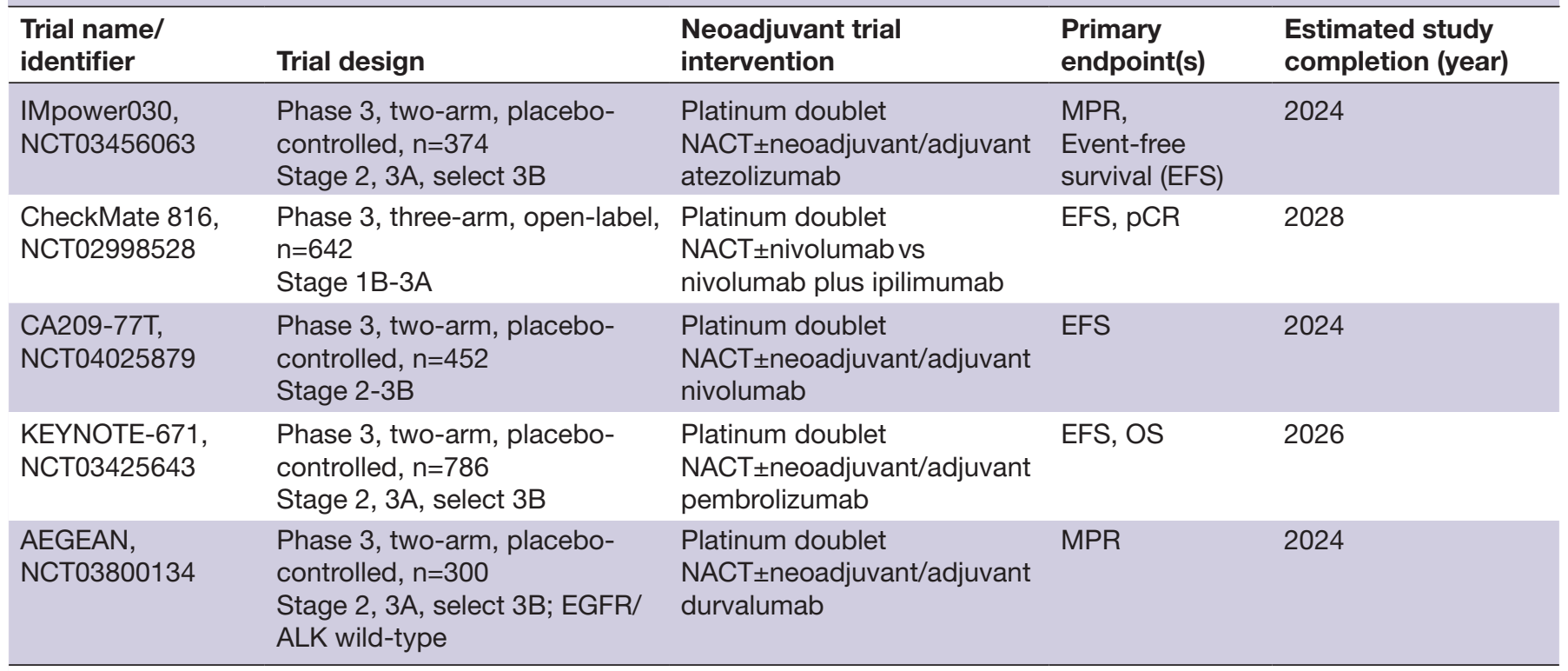

ALK, anaplastic lymphoma kinase; EFS, event-free survival; EGFR, epithelial growth factor receptor; MRP, major pathological response; NACT, neoadjuvant chemotherapy; OS, overall survival; PCR, pathological complete response.

\section{RIGHT PATIENT? BIOMARKERS OF EFFICACY IN NAICI}

Limited translational data are available to inform appropriate patient selection for these various NAICI approaches in NSCLC, but are required to optimize efficacy while minimizing excess toxicity. Pretreatment biomarkers explored in advanced NSCLC, such as PD-L1 expression or tumor mutational burden (TMB), have shown heterogeneous associations with MPR rate in NAICI trials. PD-L1 expression was significantly associated with MPR rate in NEOSTAR but was not significantly associated with MPR in LCMC3, the Forde study, or the two chemoimmunotherapy studies including NADIM. $^{38-40}$ In contrast, TMB was associated with MPR in Forde $e t$ al, but was not in LCMC3. ${ }^{38}$ More sophisticated potential biomarkers are suggested from some translational aspects of reported trials. In Forde $e t$ al, when comparing patients who achieved MPR versus no MPR, those with MPR had a more clonal T-cell population and a higher frequency of shared clones between tumor and peripheral blood. ${ }^{38}$ Overall tumor mutational burden and predicted TANA burden were both significantly correlated with degree of pathological tumor regression in this trial. ${ }^{38}$ Validating and integrating such investigations, which require sophisticated transcriptomic and in silico analysis, into the predictive algorithm when selecting appropriate patients for NAICI approaches at baseline is an emerging challenge.

Optimal methods of on-treatment efficacy assessment to guide clinical decision making also remain unclear. Changes in peripheral blood T-cell clonality after nivolumab treatment have been shown retrospectively to correlate with neoadjuvant nivolumab efficacy: increase in peripheral T-cells clones matching those within the tumor bed was correlated with pathological response. ${ }^{67}$
Assessment of response radiologically through crosssectional imaging has not correlated pathologically with NAICI response. ${ }^{38}$ The PRADO trial in stage III melanoma hypothesizes that direct assessment of NAICI response in involved clinically evident $\mathrm{LN}$ via IHC may be used to de-escalate surgical therapy in patients achieving an MPR, building on previous findings in that disease of encouraging DFS post-NAICI for responders in that setting. ${ }^{36}$ However, preoperative sampling of tumor (primary or LN) has not been part of standard NSCLC response assessment after neoadjuvant approaches, and is a more complex procedure in thoracic malignancies; it has not been employed in NAICI NSCLC trials to date.

The approach to perioperative therapy in the setting of actionable molecular genomics findings (such as EGFR mutations or ALK-rearrangement) in resectable NSCLC requires more data to define optimal standard of care. In the adjuvant setting, long-term OS follow-up for thirdgeneration EGFR tyrosine kinase inhibitor (TKI) osimertinib compared with chemotherapy is awaited from ADAURA, although a similarly designed study showing DFS benefit for the first-generation TKI gefitinib did not translate into an OS advantage. ${ }^{20}$ An adjuvant trial assessing alectinib in ALK-rearranged NSCLC is ongoing. ${ }^{70}$ A study assessing the neoadjuvant use of firstgeneration EGFR TKI erlotinib in potentially resectable stage IIIA NSCLC showed a significant improvement in PFS in the targeted therapy arm compared with chemotherapy, but a non-significant improvement in radiological response. ${ }^{71}$ The Lung Cancer Mutation Consortium LEADER trial aims to comprehensively assess neoadjuvant targeted therapies matched to oncogenic driver mutations in NSCLC (LCMC4). In contrast, PD1/PD-L1 pathway blockade has been associated with a lower chance 
of immunotherapy benefit in metastatic EGFR-mutant or ALK-rearranged NSCLC, raising a concern about whether this is a suitable strategy in the neoadjuvant setting. ${ }^{72}$

\section{RIGHT TIME? POTENTIAL PITFALLS OF IMMUNOTHERAPY GIVEN IN THE NEOADJUVANT SETTING}

Optimal timing of immunotherapy in the treatment of early-stage NSCLC remains uncertain, although large adjuvant trials including ICI in the adjuvant treatment of NSCLC are ongoing with results due in the next few years (online supplemental table 2) ${ }^{73}$ Although theoretical benefits of NAICI relate to the mechanistic nuances of these therapies, certain pitfalls and hazards have been noted thus far in NAICI trials in cancer. First, a concern with the administration of systemic therapy in general is the risk of early progression of disease rendering the patient's tumor inoperable. In LCMC3, 10 of 101 patients evaluable in the safety analysis were either found to be inoperable at exploration or had progression of disease during neoadjuvant atezolizumab resulting in abandonment of surgical plans. ${ }^{39}$ This trial permitted the enrolment of patients with stage IIIB NSCLC, and the proportion of those subsequently deemed inoperable being of more advanced stage is yet to be revealed. However, the prospect of progression during neoadjuvant treatment compromising surgical plans remains a concern. In a prior study, radiological reassessment immediately prior to surgery did not correlate with pathological response, so the optimal method of monitoring progress on NAICI is uncertain. ${ }^{38}$ Furthermore, in NEOSTAR, an 'immune flare' phenomenon was noted radiologically in LN in up to $11 \%$ patients where granuloma formation without tumor was found in the nodes pathologically. There might be a risk of avoiding curative surgery owing to concerns about disease progression. ${ }^{40}$

Second, although undue toxicity has not been noted in the NAICI NSCLC trials reported to date, the possibility that the host systemic immune system is more functional and less systemically suppressed in the setting of early compared with late cancer, ${ }^{36} 74$ potentially conveys the risk of marked immune-related-AE (irAE) development occurring concurrently with enhanced immunemediated tumor regression. In an early NAICI trial of combination ipilimumab plus nivolumab in melanoma, 18 of 20 participants $(90 \%)$ had grade $3 / 4$ irAE noted, most of which (17 of 18) required cessation of therapy. ${ }^{36}$ Interestingly, this was reminiscent of a contemporaneous trial of preoperative combination ICI in resectable stage III or oligometastatic resectable stage IV melanoma where more than $70 \%$ patients had grade 3 or higher treatment-related TRAEs. ${ }^{75}$ The high irAE rate in earlystage melanoma in the setting of combination NAICI was subsequently shown to be likely dose-related, and adjustment of ipilimumab dose in particular is likely to have decreased irAE incidence while preserving efficacy in a follow-up trial. ${ }^{76}$
Surgical comorbidity arising from NAICI treatment is another potential concern. In the pilot study of neoadjuvant nivolumab in resectable NSCLC, 14/20 surgeries were performed with an open approach, including 7/13 which were converted from an initial minimally invasive approach, related in most cases to tumor-associated inflammation and fibrosis. ${ }^{77}$ However, rates of open resection and complications were favorably compared with surgery following NACT. ${ }^{77}$ Elsewhere, lung resection for residual disease following ICI for advanced malignancy was reported as feasible although potentially technically challenging, but with acceptable complication rates. ${ }^{78}$ An analysis of surgical outcomes from TOP1201 reported no increased adverse outcomes compared with those receiving NACT alone. ${ }^{79}$ In LCMC3, only 15 of 101 participants initially planned for minimally invasive surgery required conversion to thoracotomy, with surgery following NAICI deemed feasible. ${ }^{46}$ In a phase II trial of neoadjuvant atezolizumab plus chemotherapy, $46 \%$ resections were performed via video-assisted thoracoscopic surgery with no complications attributable to neoadjuvant treatment. ${ }^{44}$

\section{FUTURE DIRECTIONS}

Neoadjuvant therapy for cancer provides a range of potential benefits, including the ability to assess on-treatment response, reduce the tumor bulk prior to surgery, and enhance tolerability. Moreover, the nature of immunotherapy provides additional theoretical rationale for neoadjuvant administration. First, given that immune checkpoint blockade enhances T-cell activation on antigen encounter, administration of ICI in the neoadjuvant setting while the primary tumor remains in situ may result in a greater breadth of T-cell responses than in the adjuvant setting after surgery. ${ }^{80}{ }^{81}$ Notably, the emergence of peripheral T-cell clones that were rarely detected in primary tumor was reported among patients receiving neoadjuvant nivolumab in NSCLC. ${ }^{38}$ Such expansion of 'subdominant' clones might reflect epitope-spreading arising from denovo priming after ICI administration, and could represent a new T-cell repertoire more ideally poised to contribute to effective antitumor immunity. ${ }^{82-84}$ Removal of the primary tumor also likely removes the risk of $\mathrm{T}$ cell exhaustion resulting from chronic antigenic exposure. In support of this, preclinical studies discussed above have shown that NAICI induces a significantly higher level of tumor antigen-specific circulating T-cells compared with the corresponding adjuvant ICI, resulting in a long-lasting effector-memory $\mathrm{T}$ cell pool. The longterm persistence of tumor antigen-specific clonal T-cells seen in animal models of effective neoadjuvant therapy, and in patients who derived durable benefit from immune checkpoint blockade in NSCLC and colorectal cancer, suggest that tumor-specific T-cell memory might be a consequence. ${ }^{28} 3785$

Unanswered questions relate to the ideal timing and duration of immunotherapy administration, including 
number of preoperative doses and scheduling with respect to surgery, predictive biomarkers to direct selection of therapy (eg, monotherapy ICI vs combination chemoimmunotherapy), and the requirement for adjuvant or 'consolidation' therapies (either chemotherapy and/or radiotherapy). Protocols to date generally recommend the standard administration of adjuvant chemotherapy or RT according to usual institutional practice following NAICI in NSCLC trials, where these have not already been given preoperatively. A concern about chemotherapy following NAICI could relate to the traditional understanding of the immunosuppressive and lymphodepleting nature of cytotoxic chemotherapy, although the interplay between chemotherapy and the immune system is nuanced. ${ }^{48}$ One preclinical study failed to demonstrate benefit of adjuvant ICI after NAICI. The PRADO expansion cohort of the OpACIN-neo trial (NCT02977052), where adjuvant ICI will be withheld in patients sustaining excellent response to NAICI, may provide insight into this question in the setting of resectable stage 3 melanoma. ${ }^{86}$ Given that ICI conveys risks of irAE that can prove lifethreatening or permanent, the identification of robust biomarkers to determine who will benefit from therapy remains a priority. As attempts are made to intensify neoadjuvant treatment, such as through ICI-ICI combinations or ICI combined with other therapies, emergence of significant AEs may be a concern. Thorough reports of safety endpoints will be crucial in the widespread acceptance of NAICI. Importantly, validating MPR as a surrogate endpoint for survival would streamline efficacy assessment and accelerate the evaluation of novel neoadjuvant approaches.

Looking forward, an area for improvement in the trial design of NAICI studies in NSCLC could relate to greater collaboration between investigators, as has been achieved in melanoma research with the International Neoadjuvant Melanoma Consortium. ${ }^{87}$ Currently, there is marked heterogeneity in trial design and endpoints across phase 2 and 3 studies, and improved collaboration to enable reasonable comparability across trials, including in the accompanying translational research, would benefit to the field.

\section{SEARCH STRATEGY}

We searched ClinicalTrials.gov for all studies in all countries relating to 'NSCLC' (condition or disease) and 'neoadjuvant' (other term) with further filters applied: recruiting (status); phase 3 (study phase). Individual trial records were then reviewed to select those employing immunotherapy in resectable disease.

\footnotetext{
Author affiliations

${ }^{1}$ School of Clinical Sciences, Monash University Faculty of Medicine Nursing and Health Sciences, Clayton, Victoria, Australia

${ }^{2}$ Medical Oncology, Monash Health, Clayton, Victoria, Australia

${ }^{3}$ Immunology in Cancer and Infection, QIMR Berghofer Medical Research Institute, Herston, Queensland, Australia

${ }^{4}$ Medical Oncology, Peter MacCallum Cancer Centre, Melbourne, Victoria, Australia
}

${ }^{5}$ Medical Oncology, Westmead Hospital, Westmead, New South Wales, Australia ${ }^{6}$ Faculty of Medicine, The University of Sydney, Sydney, New South Wales, Australia ${ }^{7}$ Medical Oncology, Royal North Shore Hospital Northern Sydney Cancer Centre, St. Leonards, New South Wales, Australia

${ }^{8}$ Medical Oncology, Princess Alexandra Hospital, Woolloongabba, Queensland, Australia

${ }^{9}$ School of Biomedical Sciences, Queensland University of Technology, Brisbane, Queensland, Australia

${ }^{10}$ Medical Oncology, Royal Brisbane and Women's Hospital, Herston, Queensland, Australia

${ }^{11}$ Faculty of Medicine, The University of Queensland, Herston, Queensland, Australia ${ }^{12}$ Medical Oncology, The Prince Charles Hospital, Chermside, Queensland, Australia

Contributors Substantial contribution to conception and design of work: all authors. Drafting the work and/or revising it critically: all authors. Final approval of the version to be published: all authors. Agreement to be accountable for all aspects of the work: all authors.

Funding The authors have not declared a specific grant for this research from any funding agency in the public, commercial or not-for-profit sectors.

Competing interests BGMH: research funding (institution): Amgen. Advisory board or consultation: Roche, AstraZeneca; Bristol-Myers Squibb, Merck Sharpe and Dohme, Pfizer, Eisai, Takeda, Boehringer-Ingelheim. BJS: Advisory board/honoraria: Roche/Genentech, AstraZeneca, Pfizer, Novartis, Bristol-Myers-Squibb, Merck, Amgen, Loxo oncology. EA: research funding (institution): Amgen. Non-financial support: Amgen, Bristol-Myers Squibb. K0: Advisory board/honoraria: Pfizer, Roche, AstraZeneca, Boehringer-Ingelheim, Bristol Myers Squibb, Merck, Lilly Oncology, Novartis, Janssen, Yuhan, Teva, Amgen and Natera; shareholder and board member: Carpe Pharmaceuticals; Carpe Vitae Pharmaceuticals; DGC Diagnostics. NP: research funding (institution): Bayer, Pfizer. Advisory board or consultation: BristolMyers Squibb, MSD, Merck-KgA, Boehringer-Ingelheim, AstraZeneca, Roche, Bayer, Novartis, Merck-Sorono, Pfizer, Takeda, Ipsen. RH: Advisory board: AstraZeneca, Bristol-Myers Squibb, Eli Lilly, Merck, Merck Sharpe and Dohme, Novartis, Oncosec Pfizer, Roche, Seagen; Speaker honorarium: Merck Sharpe and Dohme, Novartis, Roche.

Patient consent for publication Not required.

Provenance and peer review Not commissioned; externally peer reviewed.

Open access This is an open access article distributed in accordance with the Creative Commons Attribution Non Commercial (CC BY-NC 4.0) license, which permits others to distribute, remix, adapt, build upon this work non-commercially, and license their derivative works on different terms, provided the original work is properly cited, appropriate credit is given, any changes made indicated, and the use is non-commercial. See http://creativecommons.org/licenses/by-nc/4.0/.

ORCID iD

Elizabeth Ahern http://orcid.org/0000-0002-2062-5695

\section{REFERENCES}

1 Bray F, Ferlay J, Soerjomataram I, et al. Global cancer statistics 2018: GLOBOCAN estimates of incidence and mortality worldwide for 36 cancers in 185 countries. CA Cancer J Clin 2018;68:394-424.

2 Lung Cancer - Non-Small Cell: Statistics, 2020. Available: https:// www.cancer.net/cancer-types/lung-cancer-non-small-cell/statistics

3 Pignon J-P, Tribodet H, Scagliotti GV, et al. Lung adjuvant cisplatin evaluation: a pooled analysis by the lace Collaborative group. J Clin Oncol 2008;26:3552-9.

4 Strauss GM, Herndon JE, Maddaus MA, et al. Adjuvant paclitaxel plus carboplatin compared with observation in stage lb non-smallcell lung cancer: CALGB 9633 with the cancer and leukemia group $B$, radiation therapy Oncology group, and North central cancer treatment group study groups. J Clin Oncol 2008;26:5043-51.

5 Lim E, Harris G, Patel A, et al. Preoperative versus postoperative chemotherapy in patients with resectable non-small cell lung cancer: systematic review and indirect comparison meta-analysis of randomized trials. J Thorac Oncol 2009;4:1380-8.

6 Felip E, Rosell R, Maestre JA, et al. Preoperative chemotherapy plus surgery versus surgery plus adjuvant chemotherapy versus surgery alone in early-stage non-small-cell lung cancer. J Clin Oncol 2010;28:3138-45.

7 Westeel V, Quoix E, Puyraveau M, et al. A randomised trial comparing preoperative to perioperative chemotherapy in early-stage non-smallcell lung cancer (IFCT 0002 trial). Eur J Cancer 2013;49:2654-64. 
8 MacLean M, Luo X, Wang S, et al. Outcomes of neoadjuvant and adjuvant chemotherapy in stage 2 and 3 non-small cell lung cancer: an analysis of the National cancer database. Oncotarget 2018:9:24470-9.

9 Wyld L, Audisio RA, Poston GJ. The evolution of cancer surgery and future perspectives. Nat Rev Clin Oncol 2015;12:115-24.

10 Berghmans T, Durieux V, Hendriks LEL, et al. Immunotherapy: from advanced NSCLC to early stages, an evolving concept. Front Med 2020;7:90.

11 Brahmer JR, Lacchetti C, Schneider BJ, et al. Management of immune-related adverse events in patients treated with immune checkpoint inhibitor therapy: American Society of clinical oncology clinical practice guideline. J Clin Oncol 2018;36:1714-68.

12 Chiou VL, Burotto M. Pseudoprogression and immune-related response in solid tumors. J Clin Oncol 2015;33:3541-3.

13 Raz DJ, Zell JA, Ou S-HI, et al. Natural history of stage I nonsmall cell lung cancer: implications for early detection. Chest 2007;132:193-9.

14 Little AG, Rusch VW, Bonner JA, et al. Patterns of surgical care of lung cancer patients. Ann Thorac Surg 2005;80:2051-6.

15 Riaz SP, Linklater KM, Page R, et al. Recent trends in resection rates among non-small cell lung cancer patients in England. Thorax 2012;67:811-4.

16 Park S, Park IK, Kim ER, et al. Current trends of lung cancer surgery and demographic and social factors related to changes in the trends of lung cancer surgery: an analysis of the National database from 2010 to 2014. Cancer Res Treat 2017;49:330-7.

17 Ou S-HI, Zell JA, Ziogas A, et al. Prognostic factors for survival of stage I nonsmall cell lung cancer patients : a population-based analysis of 19,702 stage I patients in the California Cancer Registry from 1989 to 2003. Cancer 2007:110:1532-41.

18 Howington JA, Blum MG, Chang AC, et al. Treatment of stage I and II non-small cell lung cancer: diagnosis and management of lung cancer, 3rd ED: American College of chest physicians evidencebased clinical practice guidelines. Chest 2013;143:e278S-313.

19 Martins RG, D'Amico TA, Loo BW, et al. The management of patients with stage IIIA non-small cell lung cancer with N2 mediastinal node involvement. J Nat/ Compr Canc Netw 2012;10:599-613.

20 Herbst RS, Tsuboi M, John T, et al. Osimertinib as adjuvant therapy in patients (PTS) with stage IB-IIIA EGFR mutation positive (EGFRm) NSCLC after complete tumor resection: ADAURA. Journal of Clinical Oncology 2020;38:LBA5.

21 Douillard J-Y, Tribodet H, Aubert D, et al. Adjuvant cisplatin and vinorelbine for completely resected non-small cell lung cancer: subgroup analysis of the lung adjuvant cisplatin evaluation. $J$ Thorac Oncol 2010;5:220-8.

22 Santana-Davila R, Martins R. Treatment of stage IIIA non-small-cell lung cancer: a Concise review for the practicing oncologist. $J$ Oncol Pract 2016;12:601-6.

23 Topalian SL, Taube JM, Pardoll DM. Neoadjuvant checkpoint blockade for cancer immunotherapy. Science 2020;367:eaax0182.

24 Hellmann MD, Chaft JE, William WN, et al. Pathological response after neoadjuvant chemotherapy in resectable non-small-cell lung cancers: proposal for the use of major pathological response as a surrogate endpoint. Lancet Oncol 2014;15:e42-50.

25 Pataer A, Kalhor N, Correa AM, et al. Histopathologic response criteria predict survival of patients with resected lung cancer after neoadjuvant chemotherapy. J Thorac Oncol 2012;7:825-32.

26 Carcereny E, Martinez A, Mate JL, et al. P1.05-015 major pathological response as a predictive value of survival in early-stage NSCLC after chemotherapy: cohort of NATCH phase III trial. Journal of Thoracic Oncology 2017;12:S1983.

27 Blakely CM, McCoach CE. Role of MPR as an early signal for efficacy in neoadjuvant studies. Clin Cancer Res 2020;26:3499-500.

28 Liu J, Blake SJ, Yong MCR, et al. Improved efficacy of neoadjuvant compared to adjuvant immunotherapy to eradicate metastatic disease. Cancer Discov 2016;6:1382-99.

29 Brockwell NK, Owen KL, Zanker D, et al. Neoadjuvant interferons: critical for effective PD-1-Based immunotherapy in TNBC. Cancer Immunol Res 2017;5:871-84.

30 Brooks J, Fleischmann-Mundt B, Woller N, et al. Perioperative, spatiotemporally coordinated activation of $T$ and NK cells prevents recurrence of pancreatic cancer. Cancer Res 2018;78:475-88.

31 Cascone Tet al. Abstract 1719: superior efficacy of neoadjuvant compared to adjuvant immune checkpoint blockade in non-small cell lung cancer. Cancer Research 2018;78:1719.

32 Broz ML, Binnewies M, Boldajipour B, et al. Dissecting the tumor myeloid compartment reveals rare activating antigen-presenting cells critical for T cell immunity. Cancer Cell 2014;26:638-52.
33 Kamphorst AO, Wieland A, Nasti T, et al. Rescue of exhausted CD8 T cells by PD-1-targeted therapies is CD28-dependent. Science 2017;355:1423-7.

34 Salmon $\mathrm{H}$, Idoyaga J, Rahman A, et al. Expansion and Activation of CD103(+) Dendritic Cell Progenitors at the Tumor Site Enhances Tumor Responses to Therapeutic PD-L1 and BRAF Inhibition. Immunity 2016;44:924-38.

35 Sánchez-Paulete AR, Teijeira A, Cueto FJ, et al. Antigen crosspresentation and T-cell cross-priming in cancer immunology and immunotherapy. Ann Oncol 2017;28:xii44-55.

36 Blank CU, Rozeman EA, Fanchi LF, et al. Neoadjuvant versus adjuvant ipilimumab plus nivolumab in macroscopic stage III melanoma. Nat Med 2018;24:1655-61.

37 Liu J, Rozeman EA, O'Donnell JS, et al. Batf3 ${ }^{+}$DCs and type I IFN are critical for the efficacy of neoadjuvant cancer immunotherapy. Oncoimmunology 2019;8:e1546068.

38 Forde PM, Chaft JE, Smith KN, et al. Neoadjuvant PD-1 blockade in resectable lung cancer. N Engl J Med Overseas Ed 2018;378:1976-86.

39 Kwiatkowski DJ, Rusch VW, Chaft JE, et al. Neoadjuvant atezolizumab in resectable non-small cell lung cancer (NSCLC): interim analysis and biomarker data from a multicenter study (LCMC3). Journal of Clinical Oncology 2019;37:8503.

40 Cascone T, William WN, Weissferdt A, et al. Neoadjuvant nivolumab (N) or nivolumab plus ipilimumab (Ni) for resectable non-small cell lung cancer (NSCLC): clinical and correlative results from the NEOSTAR study. Journal of Clinical Oncology 2019;37:8504.

41 Provencio M, Nadal E, Insa A, et al. OA13.05 NADIM study: updated clinical research and outcomes. Journal of Thoracic Oncology 2019;14:S241.

42 Provencio M, Nadal E, Insa A, et al. OA01.05 phase II study of neoadjuvant Chemo/Immunotherapy for resectable stages IIIA non-smal cell lung Cancer- Nadim Study-SLCG. Journal of Thoracic Oncology 2018;13:S320.

$43 \mathrm{Yi}$ JS, Ready N, Healy P, et al. Immune activation in early-stage nonsmall cell lung cancer patients receiving neoadjuvant chemotherapy plus ipilimumab. Clin Cancer Res 2017;23:7474.

44 Shu CA, Gainor JF, Awad MM, et al. Neoadjuvant atezolizumab and chemotherapy in patients with resectable non-small-cell lung cancer: an open-label, multicentre, single-arm, phase 2 trial. Lancet Oncol 2020;21:786-95.

45 Cottrell TR, Thompson ED, Forde PM, et al. Pathologic features of response to neoadjuvant anti-PD-1 in resected non-small-cell lung carcinoma: a proposal for quantitative immune-related pathologic response criteria (irPRC). Ann Oncol 2018;29:1853-60.

46 Nyberg K. LCMC3 findings indicate neoadjuvant Atezolizumab safe, efficacious in resectable stage IB-IIIB NSCLC, 2021. Available: https://www.iaslc.org/iaslc-news/ilcn/lcmc3-findings-indicateneoadjuvant-atezolizumab-safe-efficacious-resectable-stage

47 Cascone T, William WN, Weissferdt A, et al. Neoadjuvant nivolumab or nivolumab plus ipilimumab in operable non-small cell lung cancer: the phase 2 randomized NEOSTAR trial. Nat Med 2021;27:504-14.

48 Emens LA, Middleton G. The interplay of immunotherapy and chemotherapy: harnessing potential synergies. Cancer Immunol Res 2015;3:436-43

49 Provencio M, Nadal E, Insa A, et al. Neoadjuvant chemotherapy and nivolumab in resectable non-small-cell lung cancer (NADIM): an open-label, multicentre, single-arm, phase 2 trial. Lancet Oncol 2020;21:1413-22.

50 Altorki N, Borczuk A, Saxena A, et al. P2.04-92 neoadjuvant Durvalumab with or without Sub-Ablative stereotactic radiotherapy (SBRT) in patients with resectable NSCLC (NCT02904954). Journal of Thoracic Oncology 2019;14:S746.

51 Peters S, Kim AW, Solomon B, et al. IMpower030: phase III study evaluating neoadjuvant treatment of resectable stage II-IIIB non-small cell lung cancer (NSCLC) with atezolizumab (atezo) + chemotherapy. Annals of Oncology 2019;30:ii30-30.

52 Felip E, Brahmer J, Broderick S, et al. P2.16-03 CheckMate 816: a phase 3 trial of neoadjuvant nivolumab plus ipilimumab or chemotherapy vs chemotherapy in early-stage NSCLC. Journal of Thoracic Oncology 2018;13:S831-2.

53 Fernando HC, Yang J, Ferraro GL, et al. Randomized, double-blind phase 3 study evaluating neoadjuvant platinum-based chemotherapy with perioperative pembrolizumab or placebo in resectable stage IIb or IIIA NSCLC: KEYNOTE-671. Journal of Clinical Oncology 2018;36:TPS8583.

54 Heymach J, Taube J, Mitsudomi T, et al. P1.18-02 the Aegean phase 3 trial of Neoadjuvant/Adjuvant Durvalumab in patients with resectable stage II/III NSCLC. Journal of Thoracic Oncology 2019;14:S625-6. 
55 Eichhorn F, Klotz LV, Bischoff H, et al. Neoadjuvant anti-programmed death-1 immunotherapy by pembrolizumab in resectable nodal positive stage II/IIla non-small-cell lung cancer (NSCLC): the NEOMUN trial. BMC Cancer 2019;19:413.

56 Mignard X, Antoine M, Moro-Sibilot D, et al. [loNESCO trial: Immune neoajuvant therapy in early stage non-small cell lung cancer]. Rev Mal Respir 2018;35:983-8.

57 Second affiliated Hospital, S. O. M. Z. U. (2022).

58 Gustave Roussy, C. C. G. P. (2019).

59 Neal, R., Merck, S., Dohme, C. \& Duke, U. (2019).

60 University Hospital, E. (2023).

61 Ahern E, Cubitt A, Ballard E, et al. Pharmacodynamics of preoperative PD1 checkpoint blockade and receptor activator of NFkB ligand (RANKL) inhibition in non-small cell lung cancer (NSCLC): study protocol for a multicentre, open-label, phase 1B/2, translational trial (popcorn). Trials 2019;20:753.

62 Fundación, G. (2022)..

63 Spanish lung cancer, G. (2021).

64 Swiss group for clinical cancer, R. (2021).

65 Greg Durm MD. AstraZeneca, Indiana University School of, M. \& Hoosier Cancer Research, N 2021.

66 Sidney Kimmel Comprehensive Cancer Center at Johns, H. \& AstraZeneca 2021

67 Zhang J, Ji Z, Caushi JX, et al. Compartmental analysis of T-cell clonal dynamics as a function of pathologic response to neoadjuvant PD-1 blockade in resectable non-small cell lung cancer. Clin Cancer Res 2020;26:1327-37.

68 Blank CU, Reijers ILM, Pennington T, et al. First safety and efficacy results of PRADO: a phase II study of personalized response-driven surgery and adjuvant therapy after neoadjuvant ipilimumab (IPI) and nivolumab (NIVO) in resectable stage III melanoma. Journal of Clinical Oncology 2020;38:10002.

69 Zhong W-Z, Wang Q, Mao W-M, et al. Gefitinib versus vinorelbine plus cisplatin as adjuvant treatment for stage II-IIIA (N1-N2) EGFRmutant NSCLC: final overall survival analysis of CTONG1104 phase III trial. Journal of Clinical Oncology 2021;39:713-22.

70 Solomon BJ, Ahn JS, Barlesi F, et al. ALINA: A phase III study of alectinib versus chemotherapy as adjuvant therapy in patients with stage IB-IIIA anaplastic lymphoma kinase-positive $(A L K+)$ non-small cell lung cancer (NSCLC). Journal of Clinical Oncology 2019;37:TPS8569.

71 Zhong W-Z, Chen K-N, Chen C, et al. Erlotinib Versus Gemcitabine Plus Cisplatin as Neoadjuvant Treatment of Stage IIIA-N2 EGFRMutant Non-Small-Cell Lung Cancer (EMERGING-CTONG 1103): A Randomized Phase II Study. J Clin Oncol 2019;37:2235-45.

72 Gainor JF, Shaw AT, Sequist LV, et al. Egfr mutations and ALK rearrangements are associated with low response rates to PD-1 pathway blockade in non-small cell lung cancer: a retrospective analysis. Clin Cancer Res 2016;22:4585-93.
73 Vansteenkiste J, Wauters E, Reymen B, et al. Current status of immune checkpoint inhibition in early-stage NSCLC. Ann Oncol 2019;30:1244-53.

74 Lui VK, Karpuchas J, Dent PB, et al. Cellular immunocompetence in melanoma: effect of extent of disease and immunotherapy. $\mathrm{Br} \mathrm{J}$ Cancer 1975;32:323-30.

75 Amaria RN, Reddy SM, Tawbi HA, et al. Neoadjuvant immune checkpoint blockade in high-risk resectable melanoma. Nat Med 2018;24:1649-54.

76 Rozeman EA, Menzies AM, van Akkooi ACJ, et al. Identification of the optimal combination dosing schedule of neoadjuvant ipilimumab plus nivolumab in macroscopic stage III melanoma (OpACIN-neo): a multicentre, phase 2, randomised, controlled trial. Lancet Oncol 2019;20:948-60.

77 Bott MJ, Yang SC, Park BJ, et al. Initial results of pulmonary resection after neoadjuvant nivolumab in patients with resectable non-small cell lung cancer. $J$ Thorac Cardiovasc Surg 2019;158:269-76.

78 Bott MJ, Cools-Lartigue J, Tan KS, et al. Safety and feasibility of lung resection after immunotherapy for metastatic or unresectable tumors. Ann Thorac Surg 2018;106:178-83.

79 Yang C-FJ, McSherry F, Mayne NR, et al. Surgical outcomes after neoadjuvant chemotherapy and ipilimumab for non-small cell lung cancer. Ann Thorac Surg 2018;105:924-9.

80 Wei SC, Duffy CR, Allison JP. Fundamental mechanisms of immune checkpoint blockade therapy. Cancer Discov 2018;8:1069-86.

81 O'Donnell JS, Hoefsmit EP, Smyth MJ, et al. The promise of neoadjuvant immunotherapy and surgery for cancer treatment. Clin Cancer Res 2019;25:5743-51.

82 Chen DS, Mellman I. Elements of cancer immunity and the cancerimmune set point. Nature 2017;541:321-30.

83 Memarnejadian A, Meilleur CE, Shaler CR, et al. PD-1 Blockade Promotes Epitope Spreading in Anticancer $\mathrm{CD} 8^{+} \mathrm{T}$ Cell Responses by Preventing Fratricidal Death of Subdominant Clones To Relieve Immunodomination. J Immunol 2017;199:3348-59.

84 Friedman J, Moore EC, Zolkind P, et al. Neoadjuvant PD-1 immune checkpoint blockade reverses functional immunodominance among tumor antigen-specific T cells. Clin Cancer Res 2020;26:679-89.

85 Smith KN, Llosa NJ, Cottrell TR, et al. Persistent mutant oncogene specific T cells in two patients benefitting from anti-PD-1. J Immunother Cancer 2019;7:40.

86 Reijers I, Rozeman EA, Menzies AM, et al. Personalized responsedriven adjuvant therapy after combination ipilimumab and nivolumab in high-risk resectable stage III melanoma: PRADO trial. Journal of Clinical Oncology 2019;37:TPS9605.

87 Amaria RN, Menzies AM, Burton EM, et al. Neoadjuvant systemic therapy in melanoma: recommendations of the International neoadjuvant melanoma Consortium. Lancet Oncol 2019;20:e378-89. 